\title{
Lipopolysaccharide acting via toll-like receptor 4 transactivates the TGF- $\beta$ receptor in vascular smooth muscle cells
}

\author{
Rizwana Afroz ${ }^{1,4} \cdot$ Hirushi Kumarapperuma ${ }^{1}$ Q Quang V. N. Nguyen ${ }^{1} \cdot$ Raafat Mohamed $^{1,5} \cdot$ Peter J. Little $^{1,2,3}$. \\ Danielle Kamato ${ }^{1}[$
}

Received: 31 August 2021 / Revised: 6 January 2022 / Accepted: 20 January 2022

(c) The Author(s) 2022

\begin{abstract}
Toll-like receptors (TLRs) recognise pathogen-associated molecular patterns, which allow the detection of microbial infection by host cells. Bacterial-derived toxin lipopolysaccharide activates TLR4 and leads to the activation of the Smad2 transcription factor. The phosphorylation of the Smad2 transcription factor is the result of the activation of the transforming growth factor- $\beta$ receptor 1 (TGFBR1). Therefore, we sought to investigate LPS via TLR4-mediated Smad 2 carboxy terminal phosphorylation dependent on the transactivation of the TGFBR1. The in vitro model used human aortic vascular smooth muscle cells to assess the implications of TLR4 transactivation of the TGFBR1 in vascular pathophysiology. We show that LPS-mediated Smad2 carboxy terminal phosphorylation is inhibited in the presence of TGFBR1 inhibitor, SB431542. Treatment with MyD88 and TRIF pathway antagonists does not affect LPS-mediated phosphorylation of Smad2 carboxy terminal; however, LPS-mediated Smad2 phosphorylation was inhibited in the presence of MMP inhibitor, GM6001, and unaffected in the presence of ROCK inhibitor Y27632 or ROS/NOX inhibitor DPI. LPS via transactivation of the TGFBR1 stimulates PAI-1 mRNA expression. TLRs are first in line to respond to exogenous invading substances and endogenous molecules; our findings characterise a novel signalling pathway in the context of cell biology. Identifying TLR transactivation of the TGFBR1 may provide future insight into the detrimental implications of pathogens in pathophysiology.
\end{abstract}

Keywords Smad $\cdot$ Transforming growth factor beta $\cdot$ Transactivation dependent

\section{Introduction}

Toll-like receptors (TLRs) play a critical role in bridging innate and acquired immune responses [1]. TLRs are activated by both pathogen-associated molecular

Danielle Kamato

d.kamato@uq.edu.au

1 School of Pharmacy Australia Centre of Excellence, The University of Queensland, Woolloongabba, QLD 4102, Australia

2 Department of Pharmacy, Xinhua College of Sun Yat-Sen University, Tianhe District, Guangzhou 510520, China

3 Sunshine Coast Health Institute, University of the Sunshine Coast, Birtinya, QLD 4575, Australia

4 Centre for Cancer Cell Biology and Drug Discovery, Griffith Institute for Drug Discovery, Griffith University, Nathan, Brisbane, QLD 4111, Australia

5 Department of Basic Sciences, College of Dentistry, University of Mosul, Mosul, Iraq patterns (PAMPs) and damage-associated molecular patterns (DAMPs). These receptors belong to the pattern recognition (PRR) family and underpin the pathology of numerous inflammation-related diseases, including cardiovascular disease (CVDs), diabetes and several types of cancers. The first cloned and characterised human TLR was TLR4, which is activated by lipid A, the biologically active constituent of bacterial endotoxin, lipopolysaccharide (LPS) [2]. TLRs, specifically TLR4, have an established role in multiple cardiovascular pathophysiologies [3-5], including our recent work demonstrating that LPS drives proteoglycan modifications as an initiating step in atherosclerosis [6]. LPS signalling utilises intracellular adapter molecules such as myeloid differentiation factor-88 (MyD88) and TIR domaincontaining adaptor protein (TRIP) [7]. The activation of the TLR-linked adapter molecules results in the consequent activation of downstream transcription factors NF- $\mathrm{KB}$ and AP-1. Emerging data have shown that TLR4 can also activate the Smad transcription factor [8-13]. Therefore, we sought to 
investigate the mechanism of TLR-mediated Smad signalling in human vascular smooth muscle cells (VSMCs).

Direct or canonical TGF- $\beta$ signalling commences upon the binding of the TGF- $\beta$ to the TGF receptor type II, which leads to phosphorylation and recruitment of the TGF- $\beta$ receptor type I (TGFBR1) to form a quaternary TGF- $\beta$ receptor complex that leads to the phosphorylation of the Smad transcription factor [14]. TGF- $\beta$ is produced in a 'latent' form in cells and requires activation before eliciting cellular effects. The activation of the latent TGF- $\beta$ is achieved through various mechanisms, involving integrins, proteases and reactive oxygen species (ROS) $[15,16]$. Our group identified that $\mathrm{G}$ protein-coupled receptors (GPCR) such as thrombin [17-20], endothelin [21, 22] and lysophosphatidic acid $[23,24]$ via specific biochemical mechanisms could transactivate the TGFBR 1 leading to the phosphorylation of Smad2. Transactivation-dependent signalling involves cell surface receptor transactivation of a second cell surface receptor occurring in the absence of transcription and translation of intermediate products $[25,26]$. The engagement of transactivation signalling considerably expands the range of cellular functions attributable to the first or index cell surface receptor. As an example of the quantitative contribution of transactivation signalling to the primary receptor signalling response, we have demonstrated that transactivation-dependent signalling accounts for 50\% of the total pool of genes regulated by the cognate receptor ligand and thrombin activation of protease-activated receptor (PAR)-1 [27].

The mechanisms involved in GPCR transactivation of the TGFBR1 have been characterised in detail. We have found that in VSMCs, thrombin [17-19] and lysophosphatidic acid $[23,24]$ transactivation of the TGFBR1 occurs via cytoskeletal rearrangement involving Rho-associated protein kinase (ROCK)-signalling, which leads to the activation of integrin proteins on the cell surface. Once activated, cell surface integrin induces the activation of the large latent complex (LLC) that releases TGF- $\beta$ for activation of TGFBR $1[19,28]$. The signalling paradigm described by our group was observed in several other models including lung epithelial cells [29], rat VSMCs [30] and airway smooth muscle cells [31]. The TGFBR 1 can be activated by matrix metalloproteinases (MMPs) that can cleave and liberate the TGF- $\beta$ ligand from the complex and activate downstream TGF- $\beta$ signalling [16]. The role of MMPs was assessed in GPCR transactivation of the TGFBR1 and we showed surprisingly that thrombin transactivation of the TGFBR1 was not dependent on MMPs, noting that MMP involvement in transactivation of the epidermal growth factor (EGF) receptor was one of the first documented manifestations of transactivation signalling [32].

The link between infection and cardiovascular disease has been identified; however, the mechanisms have not been fully elucidated. The identification that pathogen-sensing receptors can communicate and activate secondary cell surface receptors can explain the consequences of TLRs on several vascular pathophysiologies. TGF- $\beta$ and its cognate receptor correlate with the progression of vascular diseases, including atherosclerosis [18, 33-35]. TGFBR signalling activates multiple signalling intermediates that control the transcription of genes involved in vascular pathophysiology [36]. One such TGF- $\beta$ target gene encodes plasminogen activator inhibitor type 1 (PAI-1), a proteinase inhibitor essential for homeostasis and preclusion of induction of bleeding diathesis [37]. Increased expression of PAI-1 mRNA has been identified in various cell types, primarily localised around the base of atherosclerosis plaque [38, 39]. Administration of a PAI-1 inhibitor decreases atherosclerosis in a rodent model of obesity and the metabolic syndrome [40]. Mice express a stable form of PAI-1 in the blood that can contribute to the development of coronary artery clots without typical precursor atherosclerosis [37, 41]. Therefore, we have considered PAI-1 expression a functional readout of pathophysiologically interesting TLR4/TGFBR interactions in VSMCs.

In this study, we investigated the role of LPS and TLR4 in activating TGFBR, which was assessed by the phosphorylation of transcription factor $\mathrm{Smad} 2$ in the carboxy terminus. We characterised the signalling mechanisms by which TLR4 transactivates TGFBR in VSMCs and measured PAI-1 expression level as a functional readout of this signalling pathway.

\section{Methods and materials}

\section{Materials}

Human aortic-vascular smooth muscle cells (HA-VSMCs) (ATCC® CRL-1999) were purchased from In Vitro Technologies Life Sciences (VIC, Aus). LPS (rough strains from Salmonella enterica serotype minnesota Re 595 (Cat \#L9746)), SB431542, AG1478, SB202190, UO126 and SP600125 were purchased from Sigma-Aldrich (MO, USA). GM6001, pyridoxatin and Ab1421180 were purchased from Abcam (VIC, Australia). Human recombinant transforming growth factor $\beta 1$ (TGF $\beta 1$ ), antiphosphoSmad2 (Ser465/467), antirabbit imunoglobulinG ( $\operatorname{IgG}$ ) horseradish peroxidase (HRP) and glyceraldehyde 3-phosphate dehydrogenase (GAPDH) rabbit monoclonal IgG antibody (HRP conjugate) were purchased from Australian Bioresearch (WA, AU). Primers to $18 \mathrm{~S}$ and PAI-1were purchased from Qiagen (VIC, AUS). 


\section{Cell culture}

HA-VSMCs (passage 5-9) were grown in Ham's F-12 K (Kaighn's) medium supplemented with $10 \%$ FBS (v/v) and $1 \%$ penicillin streptomycin (v/v) at $37{ }^{\circ} \mathrm{C}$ in $5 \% \mathrm{CO}_{2}$. HAVSMCs were seeded in $60 \mathrm{~mm}$ dishes for experimentation. Cells were grown to confluence and rendered quiescent condition with Ham's F12K Ham (0.1\% FBS (v/v)) for 48 h prior the experiment.

\section{Quantitative real-time polymerase chain reaction}

The mRNA expression level of PAI-1 was determined by qRT-PCR. Total RNA was extracted from treated cells using RNeasy Mini kit (Qiagen). RNA concentration and purity were checked by spectrophotometry using Nanodrop 2000 spectrophotometer (Thermo Fisher Scientific). First-strand cDNA was synthesised from 1000 ng RNA using the Quantitect ${ }^{\circledR}$ reverse transcription kit (Qiagen). qRT-PCR was performed using Qiagen Rotor gene Q and QuantiNova ${ }^{\mathrm{TM}}$ Sybr ${ }^{\circledR}$ green PCR kit (Qiagen). The data were normalised against $18 \mathrm{~S}$ using the delta delta cycle-threshold $(\Delta \Delta \mathrm{Ct})$ method.

\section{Western blotting}

Protein isolated from treated cells was separated and transferred onto polyvinylidene difluoride membranes. Membranes were blocked for $60 \mathrm{~min}$ at room temperature with 5\% BSA and probed with anti-phospho-Smad2 (Ser465/467) (1:1000 dilution) overnight at $4{ }^{\circ} \mathrm{C}$, followed by rabbit immunoglobulin-G (IgG)- HRP secondary antibody (1:1000 dilution) for $60 \mathrm{~min}$ at room temperature. The membranes were stripped and probed with GAPDH HRP conjugated monoclonal antibody (1:4000 dilution) to determine equal loading. Enhanced chemiluminescence (ECL) was used to visualise proteins of interest. Blots were imaged with BioRad gel documentation system. Band density was measured by Image Lab imaging software (version 5.2.1). The protein of interest was normalised against housekeeping protein GAPDH.

\section{Assessment of MMP activity by gelatine zymography}

Confluent HA-VSMCs were washed with serum-free media $(0 \%$ FBS (v/v)) and then incubated for $48 \mathrm{~h}$. Following treatment, conditioned media were collected. Equal amounts of conditioned media were loaded on a $10 \%$ SDS polyacrylamide gel co-polymerised with $1 \mathrm{mg} / \mathrm{mL}$ of gelatine and electrophoresed at $120 \mathrm{~V}$ for $3 \mathrm{~h}$ on ice [42]. The gel was incubated in wash buffer (Triton X-100 2.5\% (v/v), $50 \mathrm{mM}$ Tris- $\mathrm{HCl} \mathrm{pH} 7.5$ (v/v), $5 \mathrm{mM} \mathrm{CaCl}_{2}$ (v/v)) 30 mins twice with gentle agitation. The gel was rinsed with incubation buffer (Triton X-100 1\% (v/v), 50 mM Tris-HCl pH 7.5 $(\mathrm{v} / \mathrm{v}), 5 \mathrm{mM} \mathrm{CaCl}_{2}(\mathrm{v} / \mathrm{v})$ ) for $10 \mathrm{~min}$. The gel was incubated for $24 \mathrm{~h}\left(\right.$ at $\left.37^{\circ} \mathrm{C}\right)$ in fresh incubation buffer and then stained with Coomassie brilliant blue R250 staining solution for $1 \mathrm{~h}$, followed by soaking in de-staining solution (methanol 25\% (v/v), acetic acid $10 \%(\mathrm{v} / \mathrm{v})$ and $\mathrm{H}_{2} \mathrm{O} 65 \%(\mathrm{v} / \mathrm{v})$ until clear white bands against a dark blue background appeared. The bands were detected in a ChemiDoc system and MMP activity was quantified by integrative densitometry using ImageJ (v1.52) software.

\section{Statistical analysis}

Data were normalised and presented as the mean \pm standard error of the mean (SEM). A one-way analysis of variance (ANOVA) was used to analyse statistical significance followed by least significant difference (LSD) post hoc analysis. Results were significant when the probability was less than $0.05(* p<0.05)$ and $0.01(* * p<0.01)$.

\section{Results}

\section{LPS-mediated Smad2 carboxy terminal phosphorylation is dependent on TGFBR1 activation}

LPS stimulates Smad2 carboxy-terminal phosphorylation (Ser465/467) and its nuclear translocation in cultured hepatic stellate cells (HSC-T6) [43]. We have recently reported that LPS stimulates Smad2 linker region phosphorylation in HA-VSMCs [6]; however, the role of LPS-mediated Smad2 carboxy-terminal phosphorylation in HA-VSMCs remains to be elucidated. HA-VSMCs were treated with LPS in a timedependent manner (0-240 min), and TGF- $\beta$ was used as a control. Treatment with LPS for 30 min caused a 1.4-fold increase in the phospho-Smad2 carboxy terminal (Fig. 1a) with a peak stimulation of 1.8 -fold observed at $60 \mathrm{~min}$. TGF- $\beta$, used as a control, resulted in an 11-fold increase in phospho-Smad2 carboxy terminal.

Canonical Smad2 carboxyl-terminal phosphorylation occurs as a direct outcome of the activation of the TGFBR1 [44]. To assess whether LPS signalling via its respective TLR could transactivate the TGFBR1, we utilised a pharmacological approach using the TLR4 antagonist, LPS-RS [45-47], and TGFBR1 antagonist, SB431542 [48]. LPS treatment of HA-VSMCs resulted in a 2.2-fold increase in phospho-Smad2 carboxy terminal (Fig. 1b) that was inhibited in the presence of SB431542. LPS-RS used as a control and completely inhibited LPS-mediated Smad 2 phosphorylation. The cells were treated with TGF- $\beta$ in the presence and absence of receptor antagonists, and as expected TGF- $\beta$-mediated phospho-Smad 2 


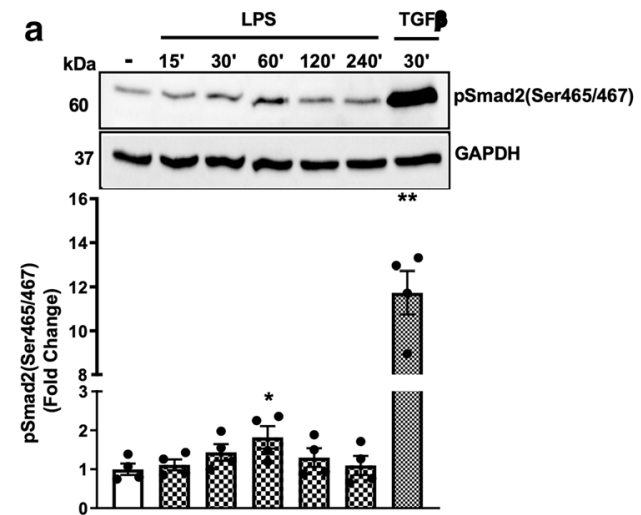

Fig. 1 LPS stimulates Smad2 carboxy terminal phosphorylation via transactivation of the TGFBR1. HA-VSMCs were treated with (a) LPS (100 ng/mL) for 0-240 min and TG-F $\beta(2 \mathrm{ng} / \mathrm{mL})$ for $30 \mathrm{~min}$ or (b) LPS $(100 \mathrm{ng} / \mathrm{ml})$ for $60 \mathrm{~min}$ or TGF $\beta(2 \mathrm{ng} / \mathrm{mL})$ for $30 \mathrm{~min}$ in the presence and absence of TGFBR1 antagonist SB431542 (SB4) $(3 \mu \mathrm{M})$ or the TLR4 inhibitor LPS-RS (RS) $(100 \mathrm{ng} / \mathrm{mL})$. Membranes were probed with monoclonal antiphosphoSmad2 (Ser465/467)

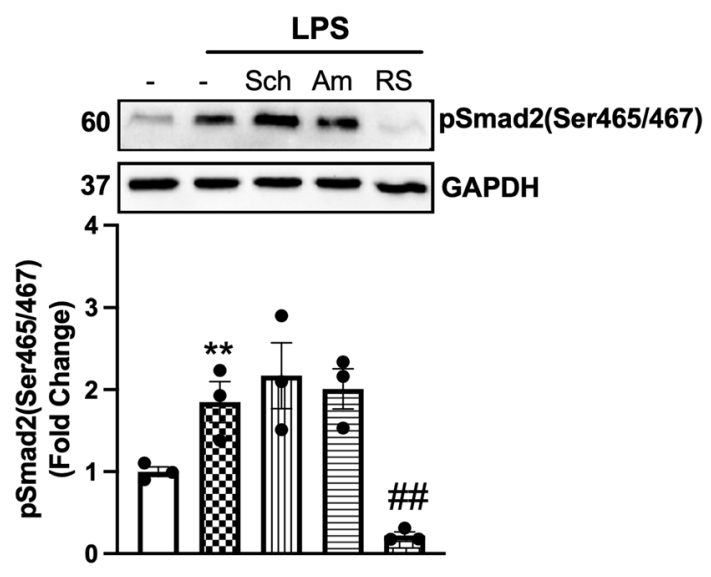

Fig. 2 TLR4 transactivation of the TGFBR1 is independent of MyD88 and TRIF-dependent pathways. HA-VSMCs were pre-incubated with schaftoside (Sch) $(10 \mu \mathrm{M})$, amlexanox (Am) $(10 \mu \mathrm{M})$ or LPS-RS (RS) (100 ng/mL), followed by LPS (100 ng/ml) for $60 \mathrm{~min}$. Membranes were probed with antiphosphoSmad2 (Ser465/467), followed by the HRP-labelled antirabbit IgG secondary, then stripped and probed with HRP-conjugated anti-GAPDH. Histograms represent band density expressed as fold change compared to no treatment control from three independent experiments. $* * p<0.01$ agonist versus untreated control and \#\#p<0.01 agonist versus antagonist using oneway ANOVA followed by LSD post hoc test

carboxy terminal was completely inhibited in the presence of SB431542 and unaffected in the presence of TLR4 antagonist (Fig. 1b). These findings demonstrate that LPS via its cognate receptor, TLR4 transactivates the TGFBR1 leading to phospho-Smad 2 carboxy terminal. These findings highlight the existence of TLR4 transactivation of the TGFBR1 in HA-VSMCS.

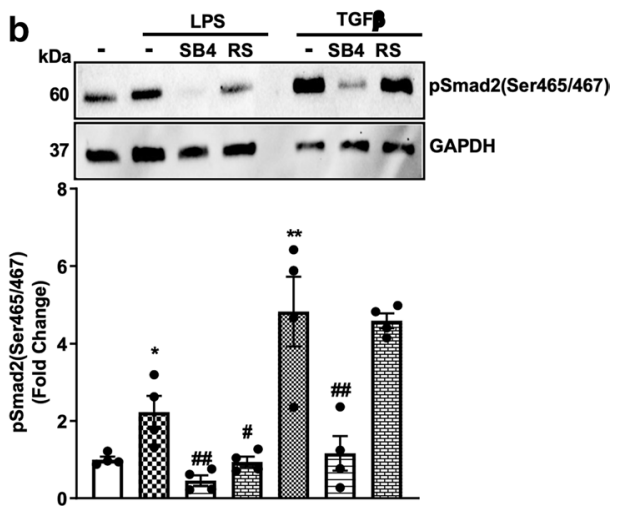

(1:1000 dilution), followed by HRP-labelled antirabbit IgG secondary (1:1000 dilution), then stripped and probed with HRP-conjugated anti-GAPDH (1:4000 dilution). Histograms represent band density expressed as fold change compared to no treatment control from four independent experiments. ${ }^{*} p<0.05$ agonist versus untreated control and $\# \# p<0.01$ agonist versus antagonist using one-way ANOVA followed by LSD post hoc test

\section{TLR4 transactivation of TGFBR 1 is independent of MyD88 and TRIF pathways}

TLR signalling pathways are classified into two distinct types: the myeloid differentiation primary response protein-88 (MyD88) and the TIR domain-containing adaptor-inducing IFN (TRIF)-dependent pathways. We asked whether TLR4 transactivation of the TGFBR1 was occurring via MyD88 or TRIF-dependent pathways (Fig. 2). Schaftoside was utilised as an inhibitor of MyD88 signalling, and amlexanox was exploited as a specific inhibitor of the TBK1-inhibiting TRIF-dependent signalling pathways. LPS-mediated phosphorylation of Smad2 carboxy terminal was unaffected in schaftoside- and amlexanox-treated cells; however, it was abolished entirely in the presence TLR4 antagonist LPS-RS. This demonstrates that the LPSmediated transactivation of the TGFBR 1 is independent of MyD88 and TRIF pathways. Therefore, we aimed to delineate the underlying mechanisms of TLR4 transactivation of the TGFBR1.

\section{TLR4 activates matrix metalloproteinases leading to phosphorylation of Smad2 carboxy terminal}

We have recently demonstrated that in HA-VSMCs, GPCR transactivation of the TGFBR1 relies on cytoskeletal rearrangement, which activates Rho/ROCK and integrin-dependent signalling leading to conformational changes in the TGF- $\beta$ complex and the activation of the TGFBR $1[23,24,32,49$, 50]. To study if LPS transactivation of the TGFBR 1 was dependent on ROCK signalling, we used the potent and selective ROCK inhibitor Y27632 (Fig. 3a). Treatment with LPS 

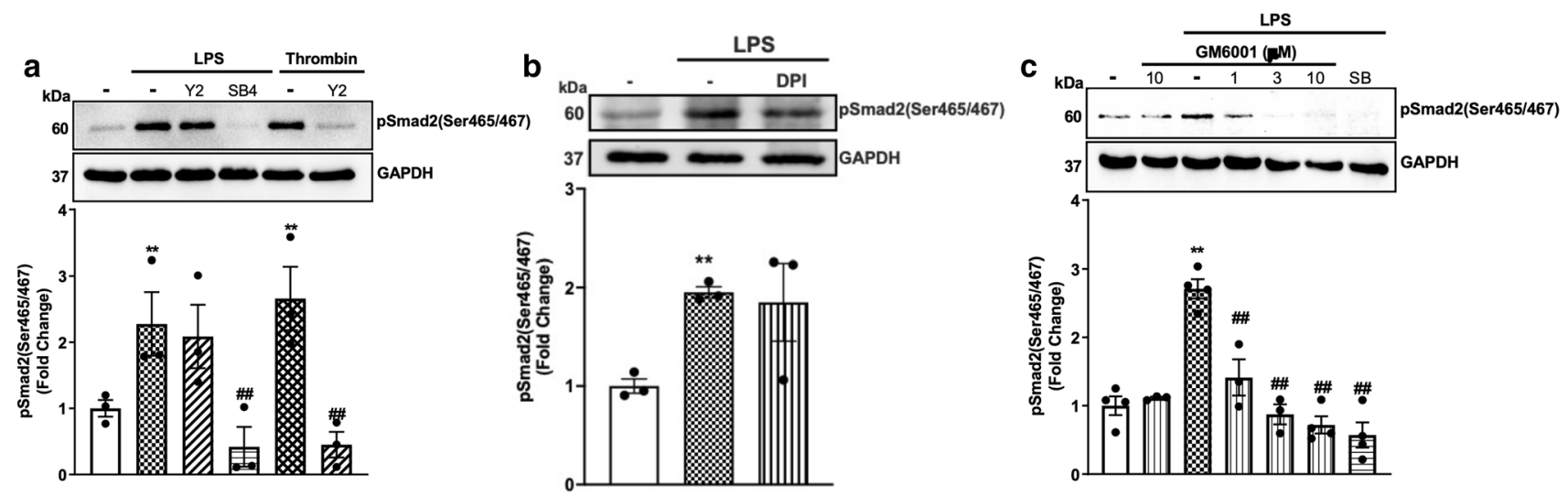

Fig. 3 TLR4 transactivation of TGFBR1 is dependent on MMPs: HA-VSMCs were treated with (a) LPS (100 ng/mL) and thrombin (10 units $/ \mathrm{mL})$ for $60 \mathrm{~min}$ in the presence and absence of ROCK inhibitor, Y27632 (Y2) $(10 \mu \mathrm{M})$ or SB431542 (SB4) $(3 \mu \mathrm{M})$ (b) ROS inhibitor, diphenyleneiodonium (DPI) or (c) preincubated with MMP inhibitor GM6001 (1-10 $\mu \mathrm{M})$ for $30 \mathrm{~min}$ followed by LPS $(100 \mathrm{ng} /$ $\mathrm{mL}$ ) for $60 \mathrm{~min}$. Membranes were probed with antiphosphoSmad2
(Ser465/467), followed by the HRP-labelled antirabbit IgG secondary, then stripped and probed with HRP-conjugated anti-GAPDH. Histograms represent band density expressed as fold change compared to no treatment control from four independent experiments. ** $p<0.01$ agonist versus untreated control and \#\#p<0.01 agonist versus antagonist using one-way ANOVA followed by LSD post hoc test

GM6001. HA-VSMCs pre-treated with SB431542 also showed complete inhibition of LPS-mediated phosphorylation of Smad 2 carboxy-terminal. These results demonstrate that TLR4 signals via MMPs to activate TGFBR1, which leads to $\operatorname{Smad} 2$ carboxy-terminal phosphorylation.

\section{MMP2, but not MMP9, is involved in LPS stimulation of phospho-Smad2 carboxy terminal}

We have identified above that MMPs are involved in LPS transactivation of the TGF- $\beta$ receptor. Specifically, MMP2, $3,9,13$ and 14 are involved in the release of TGF- $\beta$ from the TGF- $\beta$ complex. LPS increases the activity of MMP2 and MMP9 in rat aortic VSMCs [54]. We sought to investigate the role of MMP2 and MMP9 in TLR4-mediated transactivation of the TGFBR1 using a pharmacological and biochemical approach (Fig. 4a). HA-VSMCs were treated with LPS with MMP2-specific inhibitor, pyridoxatin, or MMP9-specific inhibitor, Ab1421180. Treatment with LPS increased phosphorylation of Smad 2 by 3.3 -fold that was completely inhibited in the presence of pyridoxatin and unaffected by the presence of AB1421180. We thus demonstrate that LPS signalling is via MMP2-dependent pathways to stimulate phospho-Smad2 in HA-VSMCs.

We next investigated whether LPS-mediated MMP2 secretion from HA-VSMCs (Fig. 4b). LPS treatment of HA-VSMCs resulted in a 1.7-fold increase in the secretion of pro-MMP2, and a 2.4-fold increase in the secretion of active MMP2 was observed over a 60 min treatment. These findings demonstrate that LPS stimulated MMP2 activity in HA-VSMCs, which endorses the involvement of MMPs in TLR4 transactivation of TGFBR1. 


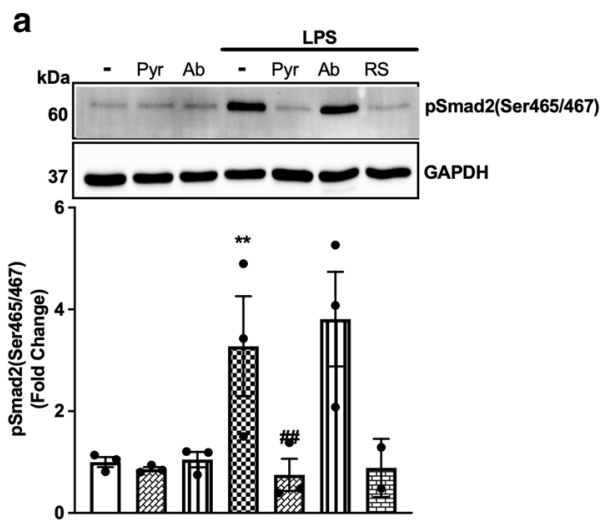

Fig. 4 LPS-mediated Smad2 carboxyl terminal phosphorylation is dependent on MMP2 activation. HA-VSMCs were preincubated with (a) pyridoxatin $(10 \mu \mathrm{M})$, Ab1421180 $(10 \mu \mathrm{M})$ or LPS-RS $(100 \mathrm{ng} /$ $\mathrm{mL})$ for $30 \mathrm{~min}$, followed by treatment with LPS $(100 \mathrm{ng} / \mathrm{mL})$ for $60 \mathrm{~min}$ (b) or treated with LPS (100 ng/ml) 30-60 min. (a) Membranes were probed with antiphosphoSmad2 (Ser465/467), followed by HRP-labelled antirabbit IgG secondary, then stripped and probed b
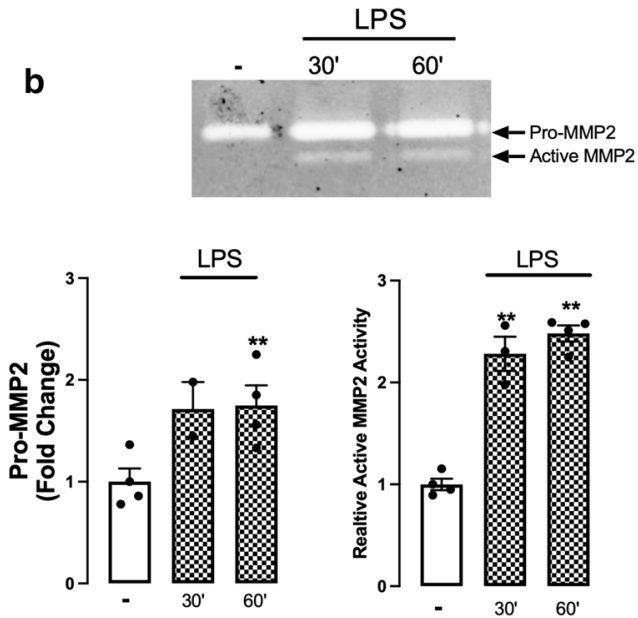

with HRP-conjugated anti-GAPDH. (b) The gelatinase activity was assessed from spent media; transparent bands were detected against a background of Coomassie brilliant blue-stained gel. Histograms represent band density expressed as fold change compared to basal from four independent experiments. $* * p<0.01$ agonist versus untreated control and \#\#p<0.01 agonist versus antagonist using one-way ANOVA followed by LSD post hoc test

a

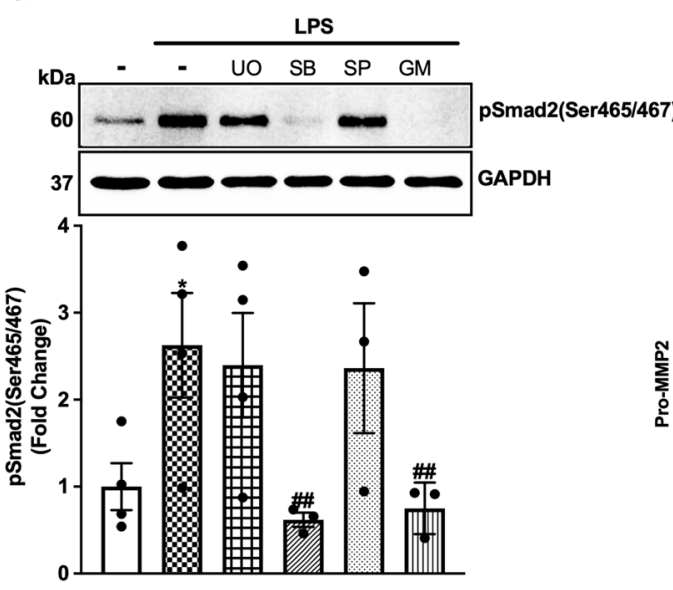

b

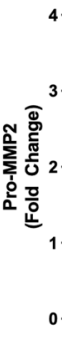

Fig. 5 MAPK p38 is involved in TLR4 transactivation of TGFBR1 in VSMCs: HA-VSMCs were pre-incubated with U0126 (UO) $(3 \mu \mathrm{M})$, SB202190 (SB) $(3 \mu \mathrm{M})$, SP600125 (SP) $(1 \mu \mathrm{M})$ and GM6001 (GM) (a) $(10 \mu \mathrm{M})($ b) $(3 \mu \mathrm{M})$ for $30 \mathrm{~min}$, followed by treatment with LPS $(100 \mathrm{ng} / \mathrm{mL})$ for $60 \mathrm{~min}$. (a) Membranes were probed with antiphosphoSmad2 (Ser465/467) followed by HRP-labelled antirabbit IgG secondary, then stripped and probed with HRP-conjugated anti-

\section{p38 via MMP2 is involved in TLR4 transactivation of TGFBR1 in VSMCs}

TGF- $\beta$-mediated Smad 2 carboxy terminal phosphorylation does not involve Erk, p38 and/or Jnk MAPKs [55]; rather, Smad 2 carboxy terminal phosphorylation is a direct readout
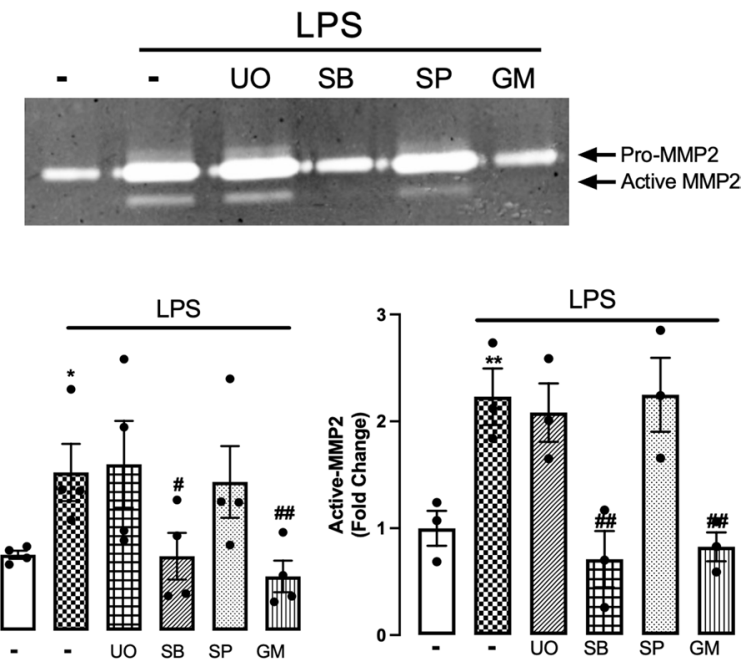

GAPDH. (b) The gelatinase activity was assessed from spent media; transparent bands were detected against a background of Coomassie brilliant blue-stained gel. Histograms represent band density expressed as fold change compared to basal from four independent experiments. Statistical significance was determined using one-way ANOVA followed by LSD post hoc test, * $p<0.05$ basal versus agonist and \#\#p $<0.01$ agonist versus antagonist

of TGFBR1 activation [56, 57]. In HSC-T6 cell lines, LPSmediated Smad2 carboxy terminal phosphorylation involves Erk, p38 and Jnk-driven pathways [43]. Here, we investigated the role of Erk, p38 and Jnk in LPS-mediated Smad2 carboxy terminal phosphorylation utilising kinase-specific pharmacological inhibitors (Fig. 5a). LPS-mediated Smad2 
phosphorylation was unaffected in the presence of Mek $1 / 2$ inhibitor, U0126, and Jnk inhibitor SP600125. In the presence of p38 inhibitor SB202190, LPS-mediated Smad2 phosphorylation was completely inhibited. MMP inhibitor GM6001 was used as a control, and completely inhibited LPS-stimulated phospho-Smad2.

We next investigated the role of MAPKs on MMP2 secretion in HA-VSMCs (Fig. 5b). LPS treatment increased the secretion of pro-MMP2 and active-MMP2 by twofold. MMP2 secretion was unaffected in the presence of U0126 and SP600125; however it was completely inhibited in the presence of p38 inhibitor SB202190. GM6001 was used as a control which showed complete inhibition of LPS-mediated MMP2 secretion. These data demonstrate that LPS via TLR4 transactivates the TGFBR1 to phosphorylate Smad2 via p38 and MMP2-dependent pathways.

\section{LPS stimulates PAI-1 mRNA expression in VSMCs via transactivation-dependent pathway}

PAI-1 plays a role in regulating the fibrinolytic system and is present in human atherosclerotic lesions. PAI-1 is markedly increased in patients with sepsis, leading to an increase in thrombus formation and severe organ dysfunction [58]. We investigated whether LPS-mediated PAI-1 expression occurs via transactivation-dependent pathways. HA-VSMCs were treated with LPS in the presence and absence of TGFBR inhibitor SB431542. We observed a 2.2-fold stimulation in PAI-1 mRNA expression (Fig. 6) that was completely inhibited in the presence of SB431542 and LPS-RS. These data demonstrate that LPS via TLR4 transactivates the TGFBR1 to stimulate PAI-1 mRNA expression in HA-VSMCs.

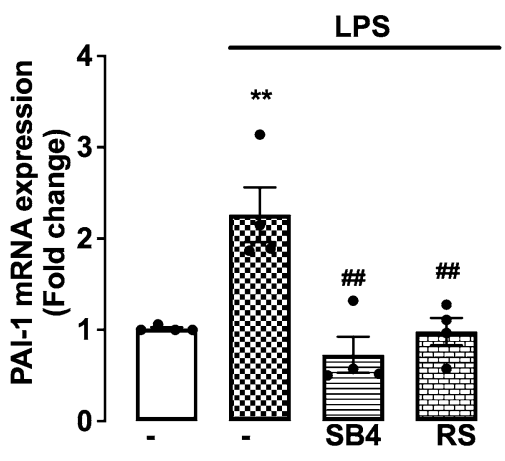

Fig. 6 Role of LPS in PAI-1 gene expression. Human VSMCs cells were treated with SB431542 $(3 \mu \mathrm{M})$ and LPS-RS $(100 \mathrm{ng} / \mathrm{mL})$ followed by treatment with LPS $(100 \mathrm{ng} / \mathrm{mL})$ for $8 \mathrm{~h}$. Total RNA was extracted and the mRNA expression of PAI-1 was analysed using qRT-PCR. Results were presented as mean \pm SEM from four separate experiments. Statistical significance was determined using one-way ANOVA followed by LSD post hoc test. $* * p<0.01$ basal versus agonist, \#\#p <0.01 agonist versus antagonist

\section{Discussion}

The engagement of receptor transactivation markedly expands the range of cellular responses attributable to the index cell surface, usually transmembrane, receptor. The current attributes of transactivation-dependent signalling encompass GPCR transactivation of kinase receptors [25, 49, 59-61]. Mechanistic studies of GPCR transactivation-dependent signalling define a concrete signalling pathway [23, 24, 32, 49]. LPS stimulates direct TGFBR1 intermediate phospho-Smad2 carboxy terminal via PI3K/ Akt and MAPK-dependent pathways in HSC-T6 hepatic stellate cells [43]. We sought out to investigate the biological mechanisms involved in TLR4-mediated Smad2 phosphorylation. We demonstrated that in HA-VSMCs, treatment with LPS increased phospho-Smad2 carboxy terminal. LPS-mediated phospho-Smad2 carboxy terminal occurs via transactivation of the TGFBR1. We characterised the TLR4 transactivation of the TGFBR1 that occurs via $\mathrm{p} 38$ activation of MMP2, which cleaves and releases the TGF- $\beta$ from the large latent TGF- $\beta$ complex. LPSmediated transactivation of the TGFBR 1 upregulated the mRNA expression of PAI-1, highlighting the significance of this novel pathway in cardiovascular complications.

TLR4 recognised by LPS interacts with three extracellular proteins: LPS binding protein, CD14 and myeloid differentiation protein 2 (MD-2). Intracellularly TLRs recruit specific adapter molecules [7]. TLR4 signalling specifically signals via MyD88 and TRIF-dependent pathways to transduce a cellular response. We demonstrate, surprisingly, that LPS-mediated transactivation of the TGFBR1 occurs independently of the well-known MyD88 and TRIF-dependent pathways. LPS-RS competes with LPS for the same binding site on MD-2; utilising LPS-RS, we demonstrate that TLR4 transactivation of the TGFBR 1 is conferred by extracellular proteins, specifically MD- 2 . Traditional activation of TLRs results in signalling via MyD88 and TRIF-dependent pathways; however, we demonstrate that TLR4 transactivation of the TGFBR1 occurs independently of these classical signalling pathways. TLR transactivation-dependent signalling represents a new signalling frontier because the identification that TLRs can transactivate other receptors greatly expands the range of signalling pathways and biological responses attributable to LPS.

In HSC-T6 hepatic stellate cells, LPS triggers the phosphorylation of Smad 2 carboxy terminal that is inhibited by the TGFBR1 antagonist [43]. However, in skin fibroblasts, treatment with LPS augments the sensitivity of fibroblasts responding to TGF- $\beta$ with a significant increase in Smad2 phosphorylation with co-treatment of LPS and TGF- $\beta$. The stimulation observed with co-treatment was completely 
lost in TLR4 silenced cells [62], which demonstrated a role for TLR4 in TGF- $\beta$-initiated signalling pathways. In contrast we demonstrate that in HA-VSMCs, TLR4-mediated phosphorylation of Smad2 occurs via the transactivation of the TGBFBR1. We also show that in TGF- $\beta$-treated cells, Smad2 phosphorylation is unaffected in the presence of competitive TLR4 antagonists LPS-RS. This demonstrates that this receptor-to-receptor communication is unidirectional in HA-VSMCs. In our studies, the cells were treated with LPS for 60 min to stimulate Smad2 phosphorylation; however, the skin fibroblasts' [62] co-exposure of LPS and TGF- $\beta$ was for $24 \mathrm{~h}$. Exposure of cells to LPS for greater than $18 \mathrm{~h}$ results in the synthesis and release of TGF- $\beta$ [63] which may have led to the augmented response observed in skin fibroblasts with co-treatment of LPS and TGF- $\beta$. Therefore, LPS via TLR4 transactivates the TGFBR1 to phosphorylate Smad2 in HA-VSMCs and HSC-T6 hepatic stellate cells [43] within a $60 \mathrm{~min}$ time frame.

Latent TGF- $\beta$ remains sequestered as part of the LLC and a variety of mechanisms that can trigger release of the TGF- $\beta$ from LLC [16]. Integrin-dependent activation of the LLC induces the release of latent TGF $\beta$ and activation of TGFBR1 in HA-VSMCs $[19,28]$. Our data showed that pharmacological inhibition of ROCK signalling utilising ROCK inhibitor, Y27632, has no effect on LPS-mediated Smad2 carboxy terminal phosphorylation. However, in the presence of Y27632, thrombin-mediated Smad2 carboxy terminal phosphorylation was completely abolished. We show that GPCR agonists thrombin [19, 32, 49] and lysophosphatidic acid [24, 64] stimulate the phosphorylation of Smad2 carboxy terminal via Rho/ROCK-dependent activation of the TGFBR1. GPCR-dependent ROCK and integrin signalling leading to the transactivation of the TGFBR1 observed in VSMCs [25] was consistent with earlier observation in mouse lung epithelial cells [29], rat VSMCs [30] and airway smooth muscle cells [31]. We also show that endogenous ROS activation of the TGFBR2 occurs via ROCK-dependent pathways [50]. Given that LPS via TLR4 transactivates the TGFBR1 independent of Rho/ROCK pathways signifies that this is a completely new signalling pathway that warrants a full investigation.

MMPs play a crucial role in releasing and activating ECM-sequestered growth and angiogenic factors, including TGF- $\beta$ [52]. MMP2 and MMP9 cleave the latent TGF- $\beta$ binding peptide to facilitate the secretion of TGF- $\beta$ in foetal rat calvaria osteoblasts [65]. Glucoseinduced phosphorylation of Smad3 in mouse embryonic fibroblasts and NRK-52E cells depended on the proteolytic release of TGF- $\beta$ from its complex [53]. Specifically, in mouse embryonic fibroblasts, the response was dependent on MMP2 and MMP9. Although several other MMPs have been identified to activate TGF- $\beta$ signalling [66, 67], MMP2 and MMP9 are the most abundantly expressed in VSMCs. We demonstrated the specificity of the response by demonstrating that MMP2, but not MMP9 is involved in TLR4 transactivation of the TGFBR1. We further showed that the release of MMP2 is dependent on the MAPK, p38. This finding is consistent with several other observations that demonstrate that silencing of $\mathrm{p} 38$ $[68,69]$ or other MAPKs $[70,71]$ regulate the release and activity of MMP2.

In a genome-wide study, GPCR transactivation-dependent signalling accounted for approximately $50 \%$ of the total genes regulated by thrombin [27]. TGF- $\beta$ plays a major role in mammalian homeostasis and is implicated in a diverse set of developmental disorders and diseases, including cancer, fibrosis, auto-immune and CVDs [72, 73] including a potent stimulator of PAI-1 expression [17, 74]. To have a functional understanding of TLR transactivation of the TGFBR1, we measured the expression of PAI-1. The main physiological action of PAI-1 is to inhibit tissue plasminogen activator and urokinase and hence inhibit fibrinolysis. PAI-1 is upregulated in endothelial cells [75], mice lung and alveolar compartment [76] when exposed to LPS. Inhalation of LPS promoted coagulation and inhibited fibrinolysis in the lungs of healthy volunteers [77]. In the vasculature, PAI-1 has several roles in the pathogenesis of ischemic heart disease [78] including stabilising the fibrin matrix for migrating cells [79, 80]. We show that LPS-mediated PAI-1 expression occurs via transactivation of the TGFBR1 in human VSMCs. Higher levels of LPS are associated with increased risk of CVDs [7]. Our results demonstrated that TLR4 activated TGFBR1 to induce the expression of PAI-1. Therefore, TLR4 transactivation of TGFBR might play a potential role in the infection-induced development and progression of atherosclerosis.

We have demonstrated that LPS via TLR4 transactivates the TGFBR1 in VSMCs via p38 and MMP2-driven pathways to stimulate the expression of PAI-1. TLRs are first in line to recognise and respond to many exogenous invading substances (pathogens) and endogenous molecules. Our findings characterise a novel signalling pathway in the context of cell biology. Identifying TLR transactivation of the TGFBR1 may provide future insight into the detrimental implications of pathogens in pathophysiology.

\section{Acknowledgements Not applicable.}

Author contributions Conceptualisation: D.K, P.J.L, R.A; data curation: R.A, R.M. H.K, Q.V.N.N; data analysis: R.A, R.M., H.K., Q.V.N.N; drafting: R.A, D.K; reviewing and editing: D.K, P.J.L.

Funding Open Access funding enabled and organized by CAUL and its Member Institutions. DK is supported by NHMRC-Peter Doherty (1160925) and National Heart Foundation (102129) fellowships. RA 
and HK were supported by The University of Queensland Research Training Programme (RTP) scholarship.

Availability of data and material The data that support the findings of this study are available from the corresponding author upon reasonable request.

\section{Declarations}

Conflict of interest The authors declare no conflict of interest.

Ethics approval and consent to participate Not applicable.

Consent for publication All authors have read and approved this draft of the manuscript.

Open Access This article is licensed under a Creative Commons Attribution 4.0 International License, which permits use, sharing, adaptation, distribution and reproduction in any medium or format, as long as you give appropriate credit to the original author(s) and the source, provide a link to the Creative Commons licence, and indicate if changes were made. The images or other third party material in this article are included in the article's Creative Commons licence, unless indicated otherwise in a credit line to the material. If material is not included in the article's Creative Commons licence and your intended use is not permitted by statutory regulation or exceeds the permitted use, you will need to obtain permission directly from the copyright holder. To view a copy of this licence, visit http://creativecommons.org/licenses/by/4.0/.

\section{References}

1. DeNardo DG, Johansson M, Coussens LM (2008) Immune cells as mediators of solid tumor metastasis. Cancer Metastasis Rev 27(1):11-18

2. Medzhitov R, Preston-Hurlburt P, Janeway CA (1997) A human homologue of the Drosophila Toll protein signals activation of adaptive immunity. Nature 388(6640):394-397

3. Mullick AE, Tobias PS, Curtiss LK (2005) Modulation of atherosclerosis in mice by Toll-like receptor 2. J Clin Investig 115(11):3149-3156

4. Michelsen KS et al (2004) Lack of Toll-like receptor 4 or myeloid differentiation factor 88 reduces atherosclerosis and alters plaque phenotype in mice deficient in apolipoprotein E. Proc Natl Acad Sci 101(29):10679-10684

5. Lehr H-A et al (2001) Immunopathogenesis of atherosclerosis. Circulation 104(8):914-920

6. Afroz R et al (2020) Toll like receptor 4 stimulates gene expression via Smad2 linker region phosphorylation in vascular smooth muscle cells. ACS Pharmacol Transl Sci 3(3):524-534

7. Zhou Y et al (2020) The Role of Toll-like Receptors in Atherothrombotic Cardiovascular Disease. ACS Pharmacol Transl Sci 3(3):457-471

8. Thuringer D et al (2011) Transactivation of the epidermal growth factor receptor by heat shock protein 90 via Toll-like receptor 4 contributes to the migration of glioblastoma cells. J Biol Chem 286(5):3418-3428

9. McElroy SJ et al (2012) Transactivation of EGFR by LPS induces COX-2 expression in enterocytes. PloS one 7(5):e38373

10. De $\mathrm{S}$ et al (2015) Erlotinib protects against LPS-induced endotoxicity because TLR4 needs EGFR to signal. Proc Natl Acad Sci 112(31):9680-9685
11. Slomiany B, Slomiany A (2016) Helicobacter pylori-elicited induction in gastric mucosal matrix metalloproteinase-9 (MMP9) release involves ERK-dependent cPLA 2 activation and its recruitment to the membrane-localized Rac1/p38 complex. Inflammopharmacology 24(2-3):87-95

12. Slomiany B, Slomiany A (2016) Helicobacter pylori-induced gastric mucosal TGF- $\alpha$ ectodomain shedding and EGFR transactivation involves Rac1/p38 MAPK-dependent TACE activation. Inflammopharmacology 24(1):23-31

13. Trussoni CE et al (2015) Lipopolysaccharide (LPS)-induced biliary epithelial cell NRas activation requires epidermal growth factor receptor (EGFR). PLoS One 10(4):e0125793

14. Rezaei HB et al (2012) Cell biology of Smad2/3 linker region phosphorylation in vascular smooth muscle. Clin Exp Pharmacol Physiol 39(8):661-667

15. Robertson IB, Rifkin DB (2016) Regulation of the bioavailability of TGF- $\beta$ and TGF- $\beta$-related proteins. Cold Spring Harbor Perspectives in Biology 8(6):a021907

16. Annes JP, Munger JS, Rifkin DB (2003) Making sense of latent TGF $\beta$ activation. J Cell Sci 116(2):217-224

17. Talati $\mathrm{N}$ et al (2018) Thrombin promotes PAI-1 expression and migration in keratinocytes via ERK dependent Smad linker region phosphorylation. Cell Signal 47:37-43

18. Burch ML et al (2010) Thrombin stimulation of proteoglycan synthesis in vascular smooth muscle is mediated by proteaseactivated receptor- 1 transactivation of the transforming growth factor beta type I receptor. J Biol Chem 285(35):26798-26805

19. Burch ML et al (2013) Thrombin-mediated proteoglycan synthesis utilizes both protein-tyrosine kinase and serine/threonine kinase receptor transactivation in vascular smooth muscle cells. J Biol Chem 288(10):7410-7419

20. Mohamed R et al (2021) Akt acts as a switch for GPCR transactivation of the TGF-beta receptor type 1. FEBS J. https://doi.org/ $10.1111 /$ febs. 16297

21. Little PJ et al (2010) Endothelin-1 stimulation of proteoglycan synthesis in vascular smooth muscle is mediated by endothelin receptor transactivation of the transforming growth factor-[beta] type I receptor. J Cardiovasc Pharmacol 56(4):360-368

22. Sharifat N et al (2017) Endothelin-1 (ET-1) stimulates carboxy terminal Smad2 phosphorylation in vascular endothelial cells by a mechanism dependent on ET receptors and de novo protein synthesis. J Pharm Pharmacol 69(1):66-72

23. Zhou $Y$ et al (2020) Lysophosphatidic acid receptor 5 transactivation of TGFBR1 stimulates the mRNA expression of proteoglycan synthesizing genes XYLT1 and CHST3. Biochim Biophys Acta Mol Cell Res 1867(12):118848

24. Zhou Y et al (2021) Curcumin Inhibits lysophosphatidic acid mediated MCP-1 expression via blocking ROCK signalling. Molecules 26(8)

25. Chaplin R et al (2017) Insights into cellular signalling by G protein coupled receptor transactivation of cell surface protein kinase receptors. J Cell Commun Signal 11(2):117-125

26. Kamato D et al (2013) Therapeutic implications of endothelin and thrombin G-protein-coupled receptor transactivation of tyrosine and serine/threonine kinase cell surface receptors. J Pharm Pharmacol 65(4):465-473

27. Kamato D et al (2017) RNA sequencing to determine the contribution of kinase receptor transactivation to $G$ protein coupled receptor signalling in vascular smooth muscle cells. PLoS One 12(7): 0180842

28. Chaplin $\mathrm{R}$ et al (2017) Insights into cellular signalling by $\mathrm{G}$ protein coupled receptor transactivation of cell surface protein kinase receptors. J Cell Commun Signal 11(2):117-125

29. Jenkins RG et al (2006) Ligation of protease-activated receptor 1 enhances alpha(v)beta6 integrin-dependent TGF-beta activation and promotes acute lung injury. J Clin Invest 116(6):1606-1614 
30. Belmadani S et al (2008) Microvessel vascular smooth muscle cells contribute to collagen type I deposition through ERK1/2 MAP kinase, alphavbeta3-integrin, and TGF-beta1 in response to ANG II and high glucose. Am J Physiol Heart Circ Physiol 295(1):H69-76

31. Tatler AL et al (2011) Integrin $\alpha v \beta 5$-mediated TGF- $\beta$ activation by airway smooth muscle cells in asthma. J Immunol 187(11):6094-6107

32. Kamato $\mathrm{D}$ et al (2016) Protease activated receptor-1 mediated dual kinase receptor transactivation stimulates the expression of glycosaminoglycan synthesizing genes. Cell Signal 28(1):110-119

33. Rostam MA et al (2018) Flavopiridol inhibits TGF-beta-stimulated biglycan synthesis by blocking linker region phosphorylation and nuclear translocation of Smad2. J Pharmacol Exp Ther. https://doi.org/10.1124/jpet.117.244483

34. Bobik A et al (1999) Distinct patterns of transforming growth factor-beta isoform and receptor expression in human atherosclerotic lesions. Colocalization implicates TGF-beta in fibrofatty lesion development. Circulation 99(22):2883-91

35. Little PJ et al (2002) Proteoglycans synthesized by arterial smooth muscle cells in the presence of transforming growth factor- $\beta 1$ exhibit increased binding to LDLs. Arterioscler Thromb Vasc Biol 22(1):55-60

36. Samarakoon R et al (2008) TGF- $\beta 1$-induced plasminogen activator inhibitor-1 expression in vascular smooth muscle cells requires pp60c-src/EGFRY845 and Rho/ROCK signaling. J Mol Cell Cardiol 44(3):527-538

37. Sobel BE, Taatjes DJ, Schneider DJ (2003) Intramural plasminogen activator inhibitor type-1 and coronary atherosclerosis. Arterioscler Thromb Vasc Biol 23(11):1979-1989

38. Schneiderman J et al (1992) Increased type 1 plasminogen activator inhibitor gene expression in atherosclerotic human arteries. Proc Natl Acad Sci 89(15):6998-7002

39. Chomiki $\mathrm{N}$ et al (1994) Plasminogen activator inhibitor-1 expression in human liver and healthy or atherosclerotic vessel walls. Thromb Haemost 72(01):044-053

40. Khoukaz HB et al (2020) Drug targeting of plasminogen activator inhibitor-1 inhibits metabolic dysfunction and atherosclerosis in a murine model of metabolic syndrome. Arterioscler Thromb Vasc Biol 40(6):1479-1490

41. Eren $M$ et al (2002) Age-dependent spontaneous coronary arterial thrombosis in transgenic mice that express a stable form of human plasminogen activator inhibitor-1. Circulation 106(4):491-496

42. Afsharimani B et al (2014) Morphine and breast tumor metastasis: the role of matrix-degrading enzymes. Clin Exp Metas 31(2):149-158

43. Kao Y-H et al (2017) Lipopolysaccharides induce Smad2 phosphorylation through PI3K/Akt and MAPK cascades in HSC-T6 hepatic stellate cells. Life Sci 184:37-46

44. Derynck R, Zhang YE (2003) Smad-dependent and Smadindependent pathways in TGF- $\beta$ family signalling. Nature 425(6958):577-584

45. Stevens CW et al (2013) Pharmacological characterization of LPS and opioid interactions at the toll-like receptor 4. Br J Pharmacol 168(6):1421-1429

46. Kutuzova GD et al (2001) Diphosphoryl lipid A from Rhodobacter sphaeroides blocks the binding and internalization of lipopolysaccharide in RAW 264.7 cells. J Immunol 167(1):482-489

47. Gaikwad S, Agrawal-Rajput R (2015) Lipopolysaccharide from Rhodobacter sphaeroides attenuates microglia-mediated inflammation and phagocytosis and directs regulatory $\mathrm{T}$ cell response. Int J Inflamm. https://doi.org/10.1155/2015/361326

48. Mori Y et al (2004) Selective inhibition of activin receptor-like kinase 5 signaling blocks profibrotic transforming growth factor $\beta$ responses in skin fibroblasts. Arthritis Rheum 50(12):4008-4021
49. Kamato D et al (2019) Mechanisms of PAR-1 mediated kinase receptor transactivation: Smad linker region phosphorylation. J Cell Commun Signal 13:539-548

50. Mohamed R et al (2020) ROS directly activates transforming growth factor beta type 1 receptor signalling in human vascular smooth muscle cells. Biochim Biophys Acta Gen Subj 1864(1):129463

51. Liu RM, Desai LP (2015) Reciprocal regulation of TGF-beta and reactive oxygen species: A perverse cycle for fibrosis. Redox Biol 6:565-577

52. Yu Q, Stamenkovic I (2000) Cell surface-localized matrix metalloproteinase-9 proteolytically activates TGF- $\beta$ and promotes tumor invasion and angiogenesis. Genes Dev 14(2):163-176

53. Wu L, Derynck R (2009) Essential role of TGF- $\beta$ signaling in glucose-induced cell hypertrophy. Dev Cell 17(1):35-48

54. Chen L, Wang W-Y, Wang Y-P (2009) Inhibitory effects of lithospermic acid on proliferation and migration of rat vascular smooth muscle cells. Acta Pharmacol Sin 30(9):1245-1252

55. Burch ML et al (2010) TGF-beta stimulates biglycan synthesis via p38 and ERK phosphorylation of the linker region of Smad2. Cell Mol Life Sci 67(12):2077-2090

56. Massagué J (2003) Integration of Smad and MAPK pathways: a link and a linker revisited. Genes Dev 17(24):2993-2997

57. Massagué J (2000) How cells read TGF- $\beta$ signals. Nat Rev Mol Cell Biol 1(3):169-178

58. Hoshino $\mathrm{K}$ et al (2017) Usefulness of plasminogen activator inhibitor-1 as a predictive marker of mortality in sepsis. J Intensive Care 5(1):42

59. Mohamed R et al (2019) GPCR transactivation signalling in vascular smooth muscle cells: role of NADPH oxidases and reactive oxygen species. Vasc Biol 1(1):R1-R11

60. Kamato D et al (2015) The expansion of GPCR transactivationdependent signalling to include serine/threonine kinase receptors represents a new cell signalling frontier. Cell Mol Life Sci 72(4):799-808

61. Dayati P et al (2018) G protein coupled receptors can transduce signals through carboxy terminal and linker region phosphorylation of Smad transcription factors. Life Sci 199:10-15

62. Bhattacharyya $S$ et al (2013) Toll-like receptor 4 signaling augments transforming growth factor- $\beta$ responses: a novel mechanism for maintaining and amplifying fibrosis in scleroderma. Am J Pathol 182(1):192-205

63. Xiao YQ et al (2008) Transcriptional and Translational Regulation of TGF- $\beta$ Production in Response to Apoptotic Cells. J Immunol 181(5):3575-3585

64. Zhou Y et al (2019) Lysophosphatidic acid and its receptors: pharmacology and therapeutic potential in atherosclerosis and vascular disease. Pharmacol Ther. https://doi.org/10.1016/j. pharmthera.2019.107404

65. Dallas SL et al (2002) Proteolysis of latent transforming growth factor- $\beta$ (TGF- $\beta$ )-binding protein- 1 by osteoclasts A cellular mechanism for release of TGF- $\beta$ from bone matrix. J Biol Chem 277(24):21352-21360

66. Niwa T et al (2018) The dynamics of TGF- $\beta$ in dental pulp, odontoblasts and dentin. Sci Rep 8(1):4450

67. Brown NF, Marshall JF (2019) Integrin-Mediated TGFbeta Activation Modulates the Tumour Microenvironment. Cancers (Basel). https://doi.org/10.3390/cancers11091221

68. Lin ML et al (2010) Down-regulation of MMP-2 through the p38 MAPK-NF-kappaB-dependent pathway by aloe-emodin leads to inhibition of nasopharyngeal carcinoma cell invasion. Mol Carcinog 49(9):783-797

69. Kumar B et al (2010) p38 mitogen-activated protein kinasedriven MAPKAPK2 regulates invasion of bladder cancer by modulation of MMP-2 and MMP-9 activity. Can Res $70(2): 832-841$ 
70. Donnini S et al (2004) ERK1-2 and p38 MAPK regulate MMP/ TIMP balance and function in response to thrombospondin-1 fragments in the microvascular endothelium. Life Sci 74(24):2975-2985

71. Xu L, Chen S, Bergan R (2006) MAPKAPK2 and HSP27 are downstream effectors of p38 MAP kinase-mediated matrix metalloproteinase type 2 activation and cell invasion in human prostate cancer. Oncogene 25(21):2987-2998

72. Kamato D et al (2013) Transforming growth factor-beta signalling: Role and consequences of Smad linker region phosphorylation. Cell Signal 25(10):2017-2024

73. Kamato D et al (2020) Smad linker region phosphorylation is a signalling pathway in its own right and not only a modulator of canonical TGF-beta signalling. Cell Mol Life Sci 77(2):243-251

74. Kamato D et al (2014) Transforming growth factor beta-mediated site-specific Smad linker region phosphorylation in vascular endothelial cells. J Pharm Pharmacol 66(12):1722-1733

75. Phillippe $\mathrm{M}$ et al (2011) Expression of coagulation-related protein genes during LPS-induced preterm delivery in the pregnant mouse. Reprod Sci 18(11):1071-1079
76. Arndt PG, Young SK, Worthen GS (2005) Regulation of lipopolysaccharide-induced lung inflammation by plasminogen activator inhibitor-1 through a JNK-mediated pathway. J Immunol 175(6):4049-4059

77. Maris NA et al (2005) Activation of coagulation and inhibition of fibrinolysis in the lung after inhalation of lipopolysaccharide by healthy volunteers. Thromb Haemost 93(06):1036-1040

78. Vaughan DE (2005) PAI-1 and atherothrombosis. J Thromb Haemost 3(8):1879-1883

79. Naito M et al (1992) Substrate-bound fibrinogen, fibrin and other cell attachment-promoting proteins as a scaffold for cultured vascular smooth muscle cells. Atherosclerosis 96(2):227-234

80. Naito M et al (1990) Effects of fibrinogen and fibrin on the migration of vascular smooth muscle cells in vitro. Atherosclerosis 83(1):9-14

Publisher's Note Springer Nature remains neutral with regard to jurisdictional claims in published maps and institutional affiliations. 